\section{Trousseau's Syndrome: Be Alert to Short-term Recurrent Cerebral Infarctions and Rapid Death}

Sir,

A 61-year male was admitted to our hospital with complaints of sudden headache, blurred vision, and visual field defects for one day. Four months prior, he was diagnosed with lung cancer and multiple lymph node metastases (Figure $1 \mathrm{~A} \& \mathrm{~B}$ ), and received targeted drug and anticoagulant therapy. However, he gradually developed bilateral pulmonary embolism, left popliteal vein thrombosis, and right intramuscular vein thrombosis. A neurologic examination revealed a hemianopia, and a grade 5 muscle strength in the left lower limb. Diffusion-weighted imaging (DWI) revealed multiple acute infarctions (Figure $1 \mathrm{C}-\mathrm{G}$ ). Magnetic resonance angiography (MRA) showed right posterior cerebral artery occlusion (Figure $1 \mathrm{H}$ ). Blood tests revealed elevated levels of fibrinogen degradation products (FDPs) of $49.34 \mathrm{ug} / \mathrm{ml}$ and D-dimers (10.37 ug/ml). He was carefully examined for an embolic stroke. However, electrocardiography, Holter, and echocardiography results were normal. Therefore, the multiple infarctions were attributed to his lung cancer (Trousseau's syndrome), and he was administered dalteparin sodium. He was admitted to hospital again 25 days after first admission for slurred speech and weakness of his right limbs. He had scattered ecchymoses on his skin, and a brain computed tomography (CT) scan showed multiple cerebral infarctions. A blood test revealed decreased platelets $\left(91 \times 10^{9} / \mathrm{L}\right)$ and higher FDPs and D-dimer levels than before; a plasma protamine paracoagulation (3P) test was also positive (the dynamic changes in FDP and D-dimer levels are shown in Figure 1I). He was diagnosed with disseminated intravascular coagulation (DIC) and died three days later.

Trousseau's syndrome is known to be a cancer-associated hypercoagulative state leading to venous and arterial thromboembolic events; ${ }^{1}$ cerebral infarctions being the most common events of arterial embolism. On magnetic resonance imaging (MRI), multiple lesions of cerebral infarction were found to be dispersed in more than two brain territories. Elevated D-dimer and FDP levels may be independent risk factors for cancer-associated cerebral infarction. ${ }^{2}$ The Ddimer levels in the acute and subacute phases were significantly higher in the cancer-related ischemic stroke group than those of the atrial fibrillation-related group. Moreover, cancerrelated ischemic stroke patients with D-dimer levels $>9.0$ $\mu \mathrm{g} / \mathrm{mL}$ had a higher probability of death within 90 days ${ }^{3}$ The Ddimer and FDP levels correlate with the effects of anticancer therapy. Early initiation of effective anticoagulant therapy is the gold standard approach to prevent thromboembolism in Trousseau's syndrome. However, Trousseau's syndrome has a poor prognosis. It is reported that the mortality rate of cancer-related stroke is up to $25-30 \%$, and more than half of the deaths occurr within 6 months after stroke. ${ }^{4}$


Figure 1: (A) Pulmonary CT showing lung cancer in the lower lobe of the right lung (arrow). (B) Hematoxylin and eosin staining showing a poorly differentiated adenocarcinoma of the lung (HE, x400). (C, D, E, F, G) Brain DWI showing fresh multiple infarctions in bilateral frontal lobes, bilateral parietal lobes, right thalamus, right temporal lobe, right occipital lobe, left temperal-occipital junction, right midbrain and bilateral cerebellar hemispheres. (H) Brain MRA showing right posterior cerebral artery occlusion. (I) A line chart showing the progressive elevation of D-dimer and FDP levels.

It is not difficult to recognise cancer-associated cerebral infarction based on MRI and blood tests, but attention should be paid to the fact that cerebral infarction can be the first clinical evidence of an occult malignancy. Moreover, once Trousseau's syndrome is diagnosed, it is necessary to closely monitor Ddimer and FDP levels to assess the efficacy of anticancer and anticoagulant therapy, and these levels can be used to analyse disease prognosis.

\section{PATIENT'S CONSENT:}

The patient and his wife consented to contribute his radiology images, hematology and pathological sections to medical research, for copyright and ethics without controversy.

\section{CONFLICT OF INTEREST:}

The authors declared no conflict of interest.

\section{AUTHORS' CONTRIBUTION:}

CZ: Reported idea and design, manuscriptwriting.

YW: Writing of manuscript, literature review.

DL: Revising it critically for important intellectual content; and final approval of the version.

\section{REFERENCES}

1. Trousseau A. Plegmasia alba dolens. Lectures on clinical medicine, delivered at the Hotel-Dieu Paris 1865; 5:281-332.

2. Kono T, Ohtsuki T, Hosomi N, Takeda I, Aoki S, Sueda Y, et al. Cancer-associated ischemic stroke is associated with elevated D-dimer and fibrin degradation product levels in 
acute ischemic stroke with advanced cancer. Geriatr Gerontol Int 2012; 12(3):468-74. doi: 10.1111/j.14470594.2011.00796.x.

3. Ito S, Kikuchi K, Ueda A. Changes in serial D-Dimer levels predict the prognoses of trousseau's syndrome patients. Front Neurol 2018; 9:528-36. doi: 10.3389/fneur.2018. 00528.

4. Zhang YY, Cordato D, Shen Q, Sheng AZ, Hung WT, Chan DK. Risk factor, pattern, etiology and outcome in ischemic stroke patients with cancer: A nested case-control study. Cerebrovasc Dis 2007; 23(2-3):181-7. doi: 10.1159/00009 7639.

Chao Zhen ${ }^{1}$, Yabo Wang ${ }^{2}$ and Dongmei $\mathrm{Li}^{1}$
${ }^{1}$ Department of Neurology, Qingdao Municipal Hospital, School of Medicine, Qingdao University, Qingdao, Shandong, P.R.

China

${ }^{2}$ Qilu Hospital (Qingdao), Cheeloo College of Medicine, Shandong University, Qingdao, Shandong, P.R. China

Correspondence to: Dr. Dongmei Li, Department of Neurology, Qingdao Municipal Hospital, School of Medicine, Qingdao University, Qingdao, Shandong 266071, P.R. China E-mail: lidongmeiqd@126.com

Received: August 24, 2020; Revised: October 31, 2020; Accepted: November 25, 2020

DOI: https://doi.org/10.29271/jcpsp.2021.08.1009 